\title{
Implicações da relação entre estigma internalizado e suporte social para a saúde: Uma revisão sistemática da literatura
}

\author{
Gabriela Correia Lubambo Ferreira \\ Universidade Federal de Juiz de Fora \\ Pollyanna Santos da Silveira \\ Universidade Federal de São Paulo \\ Universidade Federal de Juiz de Fora \\ Ana Regina Noto \\ Universidade Federal de São Paulo \\ Telmo Mota Ronzani \\ Universidade Federal de Juiz de Fora
}

\begin{abstract}
Resumo
Considerando os impactos negativos da internalização do estigma para a vida do indivíduo, e as possíveis características protetoras do suporte social para o enfrentamento da condição, objetivou-se realizar uma revisão sistemática da literatura acerca da relação entre estigma internalizado e suporte social, e suas implicações para a saúde. As buscas foram realizadas nas bases PsycInfo, Pubmed, Scopus e Web of Science. A amostra foi composta por 13 estudos que preencheram os critérios de inclusão. Os resultados apresentaram evidências de uma relação negativa entre estigma internalizado e suporte social, considerando o suporte social como uma possível estratégia de enfrentamento ao estigma internalizado, destacando o seu efeito protetor ao amenizar as consequências negativas de eventos estressantes sobre o bem-estar físico e psicológico.
\end{abstract}

Palavras-chave: estigma; suporte social; saúde.

\begin{abstract}
Implications of the relationship between internalized stigma and social support for health: A systematic review of the literature. Considering the negative impact of the internalization of stigma for the life of an individual, and the possible protective characteristics of social support for coping with the condition, the aim of this study was to conduct a systematic review of the literature about the relationship between internalized stigma and social support, and its implications for health. Searches were conducted in PsycInfo, Pubmed, Scopus and Web of Science databases. The sample consisted of 13 studies that met the inclusion criteria. Results showed evidence of a negative relationship between internalized stigma and social support, presenting social support as a possible coping strategy for internalized stigma, highlighting its protective effect to mitigate the negative consequences of stressful events on physical and psychological well-being.
\end{abstract}

Keywords: stigma; social support; health.

\section{Resumen}

Implicaciones de la relación entre el estigma internalizado y apoyo social para la salud: Una revisión sistemática de la literatu$r a$. Teniendo en cuenta los efectos negativos de la internalización de lo estigma para la vida del individuo, y las posibles características de protección de lo apoyo social para hacer frente a la situación, este artículo tiene como objetivo revisar sistemáticamente la literatura sobre la relación entre el estigma internalizado y el apoyo social y sus implicaciones para la salud. Las búsquedas se realizaron en las bases de datos PsycInfo, Pubmed, Scopus y Web of Science. La muestra consistió en 13 estudios que cumplían los criterios de inclusión. Los resultados mostraron evidencia de una relación negativa entre el estigma internalizado y el apoyo social, teniendo en cuenta el apoyo social como una posible estrategia para hacer frente al estigma internalizado, destacando sus efectos protectores para mitigar el impacto negativo de los acontecimientos estresantes en el bienestar físico y psicológico.

Palabras clave: estigma; apoyo social; salud. 
$\mathrm{O}$ conceito de doença é construído com base nos valores de vida e de saúde, representando um desvio daquilo que as pessoas esperam para si (Fife \& Wright, 2000). Algumas condições de saúde estão associadas a valores culturais que resultam na construção de uma 'identidade deteriorada', e na consequente estigmatização na medida em que elas são socialmente significativas (Fife \& Wright, 2000; Link \& Phelan, 2006). Com isso, além de precisarem enfrentar as experiências negativas decorrentes dos sintomas da própria condição de saúde, os indivíduos, muitas vezes precisam lidar com as atitudes e comportamentos negativos da sociedade, além de seus próprios (Michalak et al., 2011).

O processo de estigmatização se refere à desvalorização de um indivíduo com base em características físicas ou pessoais que ele possui, consideradas socialmente inaceitáveis, associadas a reações negativas, julgamentos morais e discriminação (Corrigan \& Watson, 2002). Entre as diversas formas prejudiciais do estigma, destaca-se o estigma internalizado e seu efeito na qualidade de vida dessas pessoas (Corrigan et al., 2010; Corrigan, Sokol, \& Rüsch, 2011). A internalização do estigma consiste em um processo subjetivo que ocorre quando membros de um subgrupo estigmatizado concordam com os preconceitos associados à sua condição, e aplicam essas atitudes e crenças negativas a si mesmo (Corrigan \& Watson, 2002). Ao se tornar consciente dos estereótipos negativos associados à sua condição de saúde, o indivíduo tende a antecipar a rejeição, desvalorização e discriminação dos outros e, então, passa a desenvolver estratégias para prevenir essas experiências, entre elas a evitação das interações sociais, e a ocultação da condição de saúde e do histórico pessoal de tratamento (Mueller et al., 2006). Além disso, essas pessoas sofrem, ainda, com baixa autoestima e baixa autoeficácia (Corrigan, Watson, \& Barr, 2006; Watson, Corrigan, Larson, \& Sells, 2007), o que leva a menor satisfação com domínios importantes da vida incluindo o trabalho, casa, espiritualidade e relacionamentos interpessoais (Corrigan et al., 2011).

Há evidências de que um bom suporte social pode atuar como fator de proteção (Chou, Robb, Clay, \& Chronister, 2013; Walton, Blow, Bingham, \& Chermack, 2003), enquanto que uma rede social pobre pode contribuir para a vulnerabilidade, para internalização de atitudes estigmatizantes, além de fazer com que os indivíduos percebam mais fortemente a desvalorização e a discriminação (Sibitz et al., 2011). Além disso, o medo da rejeição em si pode levar a percepções deturpadas do suporte e das interações sociais disponíveis, assim como também pode aumentar a exclusão por parte de familiares e amigos (Mickelson, 2001).

O construto suporte social pode ser definido como o sentimento e a percepção de estima e de pertencimento a uma rede social com direitos e deveres comuns. Refere-se à função e qualidade das relações sociais, bem como a percepção de disponibilidade de ajuda, ou suporte recebido de fato (Schwarzer \& Knoll, 2007; Seidl \& Tróccoli, 2006). Measelle, Stice e Springer (2006) destacam que à medida que o indivíduo pode contar com um suporte satisfatório aumentam as chances de ele se sentir aceito e valorizado em seu ambiente social, e assim, aumentar sua autoestima, autoeficácia e a confiança em si próprio. Mueller et al.(2006) ressaltam que a percepção de estigmatização é altamente influenciada pelo quanto as pessoas percebem as próprias relações sociais como fontes de suporte. Dessa forma, estudos têm discutido o construto suporte social como mediador do impacto do estigma na saúde dos indivíduos, atuando como um fator importante para o ajustamento físico e psicológico, oferecendo ferramentas para lidar com os estressores de vida diários (Chou et al., 2013; Mickelson, 2001; Muller et al., 2006; Vyavaharkar et al., 2010).

Tendo em vista o potencial impacto negativo da internalização do estigma para a saúde e os possíveis efeitos protetores do suporte social no enfrentamento da condição (Corrigan \& Watson, 2002; Sibitz, et al., 2011), considera-se fundamental analisar e sintetizar a produção científica na área de forma que as evidências possam vir a auxiliar no planejamento e desenvolvimento de estratégias de enfrentamento ao estigma, bem como no delineamento de pesquisas futuras. Assim, o presente estudo teve como objetivo realizar uma revisão sistemática da literatura acerca da relação entre estigma internalizado e suporte social, e analisar as implicações dessa relação para a saúde.

\section{Método}

A revisão sistemática consiste em uma revisão de uma pergunta claramente formulada que usa métodos sistemáticos e explícitos para identificar, selecionar e avaliar criticamente pesquisas relevantes, e coletar e analisar dados dos estudos selecionados (Cochrane Collaboration, 2013).

As buscas foram realizadas em quatro bases de dados a fim de ampliar o escopo da pesquisa, sendo elas PsycInfo, Pubmed, Web of science e Scopus, através das palavras-chave "self-stigma" ou "internalized stigma" que foram cruzadas com o termo "social support" utilizando o operador booleano "AND". As buscas foram realizadas desde a primeira publicação identificada nas bases até Setembro de 2012.

Para compor a amostra deste estudo, foram definidos os seguintes critérios de inclusão: (a) ser um estudo empírico; (b) apresentar a relação entre estigma internalizado e suporte social entre portadores de condições de saúde no resumo do artigo; (c) ter o texto completo disponibilizado on-line para leitura ou através do contato direto com o autor; (d) ter sido publicado em inglês, espanhol ou português. No caso de discordância acerca da classificação dos artigos, seria feita uma discussão entre os avaliadores para se chegar a um consenso final. Com este delineamento, 13 artigos constituíram a amostra final do presente estudo. A estratégia de busca dos estudos para a realização desta revisão sistemática encontra-se descrita na Figura 1. 


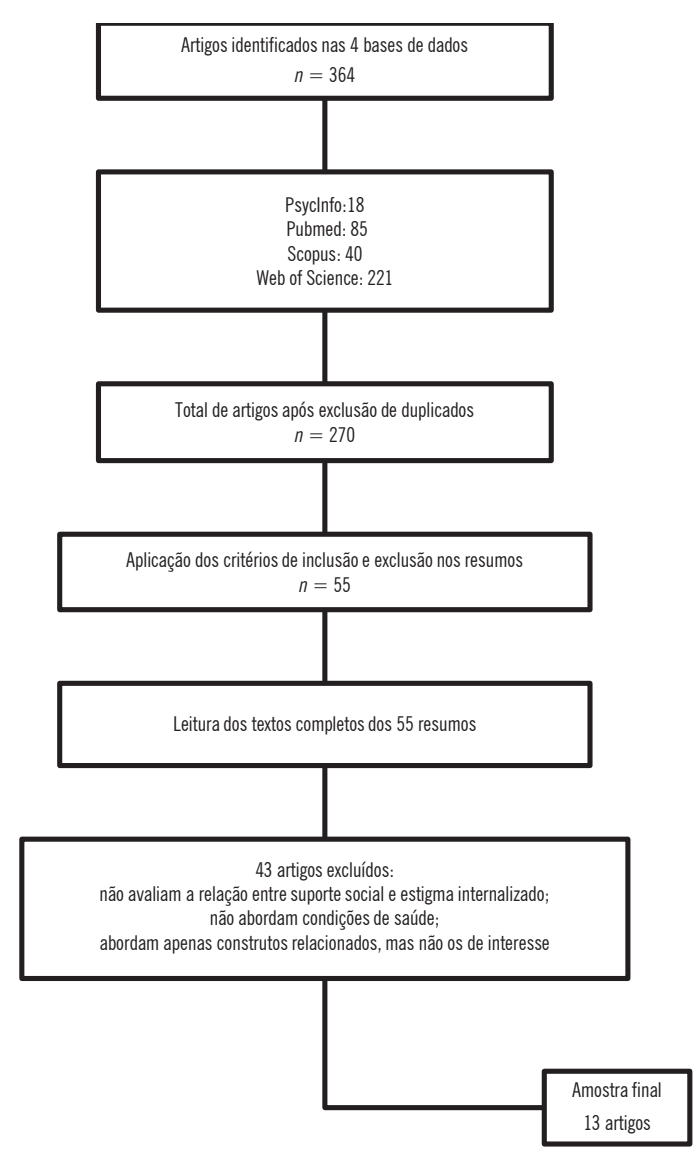

Figura 1: Estratégia de Busca nas Bases de Dados

A fim de caracterizar a produção científica na área, os seguintes indicadores bibliométricos foram analisados: autores, ano, revistas de publicação e país de estudo. Em seguida, realizou-se uma análise qualitativa dos textos completos cujos resultados foram descritos nas seguintes categorias: introdução dos estudos, desenho dos estudos, principais resultados, principais limitações e direcionamentos para pesquisas futuras.

A categoria introdução dos estudos avalia se o foco do estudo consiste na relação entre estigma internalizado e suporte social a partir dos objetivos propostos, e se estes construtos foram bem definidos pelos autores. Quanto ao desenho dos estudos, foi analisada a natureza do estudo, os métodos de amostragem, descrição dos critérios de elegibilidade e população estudada e a natureza dos instrumentos utilizados. $\mathrm{Na}$ categoria principais resultados dos estudos foi avaliada a descrição dos resultados da relação entre estigma internalizado e suporte social. Por fim, na categoria principais limitações $e$ direcionamentos para pesquisas futuras foi avaliada a apresentação das principais limitações dos estudos e sugestões para pesquisas futuras.

\section{Resultados}

\section{Indicadores bibliométricos}

No que se refere à autoria dos artigos, todos os estudos foram classificados como autoria múltipla, ou seja, escritos em colaboração com outros autores, com mínimo de dois e máximo de seis autores por estudo. Encontrou-se uma diversidade de autores que publicaram sobre o tema, com 52 autores publicando apenas uma vez. Apenas três autores publicaram duas vezes cada na área, sendo eles: Aallanise Cloete, Leickness C. Simbayi e Seth C. Kalichman.

Em relação às revistas de publicação, observou-se que 10 revistas apresentavam artigos publicados na área, sendo que oito delas publicaram apenas uma vez. A revista com maior número de publicações foi a Social Science \& Medicine com três publicações, seguida pela AIDS Care com duas publicações. A fim de assegurar um padrão de qualidade dos estudos, todas as revistas seguiram o processo de revisão por pares dos artigos.

As primeiras publicações sobre a relação entre estigma internalizado e suporte social foram em 2007 com três artigos publicados. No ano de 2008 foi publicado apenas um artigo, sendo que em 2009, 2010 e 2011 foram publicados dois artigos por cada ano. Até a data da busca, três estudos haviam sido publicados no ano de 2012.

Considerando os países em que os estudos foram desenvolvidos, três foram realizados nos Estados Unidos, três na África do Sul e três na Turquia, seguidos por dois estudos que foram realizados na China. Os demais países $(n=24)$ tiveram apenas um estudo desenvolvido na região, sendo eles: Bélgica, Bulgária, Canadá, Croácia, Eslovênia, Espanha, Estônia, Finlândia, França, Gana, Grécia, Itália, Lituânia, Macedônia, Malta, Nigéria, Polônia, República Tcheca, República Dominicana, Romênia, Rússia, Suazilândia, Suécia e Ucrânia. Ressalta-se que o estudo de Brohan, Elgie, Sartorius, Thornicroft e GAMIAN-Europe Study Group (2010) foi desenvolvido com amostras de 20 países europeus.

Análise qualitativa dos estudos

Introdução dos estudos. A partir da leitura dos objetivos dos estudos observou-se que o estudo da relação entre estigma internalizado e suporte social constituiu um dos focos principais de investigação em apenas quatro estudos (Brohan, Elgie, Sartorius, Thornicroft, \& GAMIAN-Europe Study Group, 2010; Mak et al., 2007; Peltzer \& Ramlagan, 2011; Vyavaharkar et al., 2010). Os demais nove estudos apresentaram dados dessa relação como um resultado secundário da pesquisa (Adewuya, Owoeye, Erinfolami, \& Ola, 2011; Cerit, Filizer, Tural, \& Tufan, 2012; Ersoy \& Varan, 2007; Kalichman et al., 2009; Lv, Wolf \& Wang, 2012; Person, Bartholomew, Gyapong, Addiss, \& van den Borne, 2009; Sayles et al., 2008; Simbayi et al., 2007; Zafran, Tallant, \& Gelinas, 2012).

Em relação à definição dos conceitos das variáveis estigma internalizado e suporte social, observou-se que houve uma grande variedade de autores referenciados para a de nição de estigma internalizado, embora o conceito tenha sido definido 
de forma semelhante. O principal autor referenciado foi $\mathrm{Pa}$ trick Corrigan, que argumenta que o estigma internalizado ocorre a partir da consciência dos preconceitos, estereótipos e discriminação por parte da sociedade em relação à condição de saúde, resultando na aplicação desses estereótipos negativos a si próprio, tendo como consequência a internalização da vergonha, culpa, desesperança e afastamento do convívio social em função do medo da discriminação por parte dos outros (Corrigan \& Watson, 2002). O estudo de Brohan et al. (2010) complementa ainda que a internalização do estigma é um processo de transformação de identidade, em que o indivíduo passa a adotar uma visão estigmatizada sobre si mesmo, definição proposta por Yanos, Roe, Markus e Lysaker (2008). Seis estudos não apresentaram nenhuma definição do conceito de estigma internalizado.

Já no que se refere ao conceito de suporte social, nenhum dos 13 estudos analisados apresentaram uma definição do construto, o que indica a falta de consolidação de uma definição de suporte social na literatura. Ainda, os autores não esclarecem quais as dimensões do suporte social estão sendo investigadas nos estudos.

Desenho dos estudos. Do total de 13 estudos, 11 apresentaram um delineamento transversal e apenas dois estudos foram caracterizados como longitudinais (Mak et al., 2007; Peltzer \& Ramlagan, 2011). A metodologia quantitativa de análise de dados foi utilizada em 11 estudos, os outros dois (Person et al., 2009; Zafran et al., 2012) optaram pelo desenho qualitativo.

Quanto à forma de recrutamento dos participantes, a maioria dos estudos ( $n=8$ ) optou por amostragem não-probabilística, sendo que destes, sete estudos tiveram amostras por conveniência (Ersoy \& Varan, 2007; Kalichman et al., 2009; Lv et al., 2012; Mak et al., 2007; Sayles et al., 2008; Simbayi et al., 2007; Vyavaharkar et al., 2010), enquanto apenas um estudo optou pela amostragem intencional de caso típico (Zafran et al., 2012). Quanto aos demais, três estudos optaram pela amostragem probabilística, sendo que dois pelo método de amostragem aleatória simples (Adewuya et al., 2011; Brohan et al., 2010) e um pelo método de amostragem sistemática (Peltzer \& Ramlagan, 2011). Dois estudos não relataram a forma de recrutamento de seus participantes (Cerit et al., 2012; Person et al., 2009). Ainda assim, os critérios de elegibilidade para participação no estudo estiveram bem definidos em apenas oito estudos (Adewuya et al., 2011; Brohan et al., 2010; Cerit et al., 2012; Lv et al., 2012; Peltzer \& Ramlagan, 2011; Person et al., 2009; Sayles et al., 2008; Vyavaharkar et al., 2010).

Ressalta-se que as principais populações estudadas foram pessoas que vivem com HIV/AIDS $(n=6)$ e pacientes com transtorno mental $(n=6)$, sendo a esquizofrenia o principal transtorno abordado. O estudo de Person et al. (2009) investigou o estigma internalizado entre mulheres com filariose linfática com linfedemas na perna.

Para a operacionalização do conceito de estigma internalizado, o instrumento mais utilizado foi a escala Internalized
Stigma of Mental Illness (ISMI) (Ritsher, Otilingam, \& Grajales, 2003) presente em cinco estudos. Os demais estudos quantitativos optaram pela escolha de medidas já consolidadas na literatura, enquanto que apenas o estudo de Mak et al. (2007) optou pela utilização de uma mensuração própria de estigma internalizado. Com relação à mensuração do suporte social, observou-se uma ampla variação de instrumentos destinados a avaliar esse construto, sendo que o mais utilizado foi o Social Support Questionnaire (SSQ) (Brock, Sarason, Sarason, \& Pierce, 1996) presente em três estudos. Apenas dois estudos optaram pela utilização de um instrumento próprio de suporte social (Adewuya et al., 2011; Brohan et al., 2010).

Principais resultados dos estudos. Ao avaliar a relação específica entre estigma internalizado e suporte social descrita pelos estudos, observou-se que todos os 13 artigos destacaram uma relação negativa entre essas variáveis, ou seja, níveis maiores de estigma internalizado estiveram associados a um baixo suporte social. Entre os quatro estudos que encontraram um baixo suporte social como um dos preditores de altos níveis de estigma internalizado (Adewuya et al., 2011; Brohan et al., 2010; Lv et al., 2012; Peltzer \& Ramlagan, 2011), destacou-se a importância do investimento nas relações sociais como um fator potencial no enfrentamento do estigma, devendo ser abordado no tratamento de pessoas com transtorno mental e pessoas que vivem com HIV/AIDS. Ainda, no que se refere aos efeitos protetores do suporte social, Zafran, Tallant e Gelinas (2012) encontraram que maior suporte social esteve associado com maior engajamento em atividades diárias, crença na capacidade pessoal e maior aceitação dos limites da condição de saúde para uma melhor reinserção no ambiente social entre pacientes com transtorno mental.

Já os estudos de Vyavaharkar et al. (2010) e de Simbayi et al. (2007) revelaram que altos níveis de estigma internalizado e baixo suporte social atuam como preditores significativos de depressão entre pessoas que vivem com HIV/AIDS e de funcionamento pobre de pacientes com transtorno bipolar, como descrevem Cerit, Filizer, Tural e Tufan (2012). Vyavaharkar et al. (2010) discutem ainda que o estigma internalizado pode influenciar a avaliação que os indivíduos fazem das fontes de suporte social disponíveis, sendo que uma avaliação negativa poderia atuar como preditora significativa de depressão.

O estudo de Person et al. (2009) realizado com mulheres portadoras de filariose linfática destacou que a presença dos linfedemas na perna resultam em atitudes prejudiciais e discriminatórias que, por sua vez, geram a internalização do estigma e uma incerteza nas relações sociais, o que pode contribuir para a evitação e isolamento social. Mak et al. (2007) destacaram que o estigma internalizado tem um efeito altamente negativo no suporte social de pessoas que vivem com HIV/AIDS, o que pode levar a experiências de angústia psicológica pelos indivíduos.

Os estudos de Ersoy e Varan (2007), Sayles et al. (2008) e Kalichman et al. (2009) consistiram na avaliação das propriedades psicométricas de instrumentos para avaliação do 
estigma internalizado, utilizando medidas de suporte social como critério para validade discriminante, relatando uma correlação negativa entre as variáveis. Ainda, o estudo de Ersoy e Varan (2007) considera que a qualidade dos relacionamentos sociais pode influenciar na internalização dos estereótipos e julgamentos negativos acerca do transtorno mental, na medida em que a redução dessas interações sociais prevê a internalização do estigma.

Considerando o processo de internalização do estigma, variáveis sociodemográficas como desemprego (Adewuya et al., 2011; Brohan et al., 2010; Lv et al., 2012), baixa renda (Ersoy \& Varan, 2007; Peltzer \& Ramlagan, 2011; Sayles et al., 2008;), ser do sexo masculino (Ersoy \& Varan, 2007; Simbayi et al., 2007), maiores anos de duração da doença (Adewuya et al., 2011; Simbayi et al., 2007), e baixa escolaridade (Brohan et al., 2010; Sayles et al., 2008) estiveram associadas a maiores níveis de estigma internalizado. Em relação ao suporte social, o sexo foi a principal variável sociodemográfica associada a maior nível de suporte social, o que significa que mulheres tendem a perceber maior apoio social do que homens (Cerit et al., 2012; Simbayi et al., 2007).

Quanto às variáveis psicossociais, a perda e restrição das interações sociais (Adewuya et al., 2011; Brohan et al., 2010; Ersoy \& Varan, 2007; Peltzer \& Ramlagan, 2011; Person et al., 2009), angústia psicológica (Mak et al., 2007; Person et al., 2009; Simbayi et al., 2007) e presença de sintomas depressivos (Peltzer \& Ramlagan, 2011; Simbayi et al., 2007; Vyavaharkar et al., 2010) foram considerados os principais fatores de risco para a internalização do estigma, trazendo implicações para a qualidade de vida dos indivíduos (Peltzer \& Ramlagan, 2011; Person et al., 2009). Já em relação ao suporte social, o bem-estar psicológico foi considerado a variável associada a maiores níveis de percepção de suporte (Mak et al., 2007). As descrições das características dos estudos e dos principais resultados estão sumarizadas na Tabela 1 .

Tabela 1

Descrição das Características e Principais Resultados dos Estudos.

\begin{tabular}{|c|c|c|c|c|c|}
\hline Referência & Objetivo & $\begin{array}{l}\text { Tamanho da } \\
\text { Amostra (n); } \\
\text { População }\end{array}$ & $\begin{array}{l}\text { Instrumentos } \\
\text { Estigma } \\
\text { Internalizado }\end{array}$ & $\begin{array}{l}\text { Instrumentos } \\
\text { Suporte Social }\end{array}$ & Principais Resultados \\
\hline Lv, et al. (2012) & Não está claramente descrito no artigo. & $\begin{array}{l}\quad \mathrm{n}=95 \\
\text { Esquizofrenia }\end{array}$ & ISMI & SSRS & $\begin{array}{l}0 \text { estigma internalizado foi negativamente correlacionado com } \\
0 \text { suporte social. Os autores destacam que } 0 \text { suporte social teria } \\
\text { efeito protetor no enfrentamento ao estigma. }\end{array}$ \\
\hline Cerit et al. (2012) & $\begin{array}{l}\text { Investigar os preditores de funcionamen- } \\
\text { to, como variáveis demográficas e clíni- } \\
\text { cas, suporte social, estigma internalizado } \\
\text { e insight entre pacientes com transtorno } \\
\text { bipolar em remissão dos sintomas. }\end{array}$ & $\begin{array}{l}n=80 \\
\text { Transtorno } \\
\text { Bipolar }\end{array}$ & ISMI & MSPSS & $\begin{array}{l}\text { Correlação negativa entre estigma internalizado e suporte social } \\
\text { percebido. Altos níveis de estigma internalizado e baixo suporte so- } \\
\text { cial percebido foram indicadores de um pobre funcionamento entre } \\
\text { os pacientes com transtorno bipolar. }\end{array}$ \\
\hline $\begin{array}{l}\text { Zafran, et al. } \\
\text { (2012) }\end{array}$ & $\begin{array}{l}\text { Explorar a experiência de adultos jovens } \\
\text { retornando aos estudos após um episó- } \\
\text { dio psicótico nos } 5 \text { anos anteriores. }\end{array}$ & $\begin{array}{l}\mathrm{n}=5 \\
\text { Transtorno } \\
\text { mental }\end{array}$ & Não apresenta & Não apresenta & $\begin{array}{l}\text { Efeitos positivos da presença de fontes de suporte social para } \\
\text { fornecer um ambiente seguro, facilitando o engajamento nas ati- } \\
\text { vidades diárias, ajudando a normalizar a situação de estresse e } \\
\text { vulnerabilidade associada ao transtorno mental. }\end{array}$ \\
\hline $\begin{array}{l}\text { Peltzer \& Ramla- } \\
\text { gan (2011) }\end{array}$ & $\begin{array}{l}\text { Avaliar o estigma internalizado entre } \\
\text { pessoas que vivem com HIV/AIDS ao lon- } \\
\text { go de } 1 \text { ano após o início do tratamento } \\
\text { com a terapia antirretroviral. }\end{array}$ & $\begin{array}{l}\mathrm{n}=735 \\
\text { HIV/AIDS }\end{array}$ & $\begin{array}{l}\text { AIDS-related } \\
\text { stigma }\end{array}$ & SSQ & $\begin{array}{l}\text { A falta de suporte social estava associada a maiores níveis de es- } \\
\text { tigma internalizado. Altos níveis de estigma internalizado estiveram } \\
\text { associados com depressão e baixa qualidade de vida. }\end{array}$ \\
\hline $\begin{array}{l}\text { Adewuya et al. } \\
\text { (2011) }\end{array}$ & $\begin{array}{l}\text { Avaliar a extensão, os domínios e cor- } \\
\text { relações do estigma internalizado entre } \\
\text { pacientes psiquiátricos de Lagos, Nigéria }\end{array}$ & $\begin{array}{l}\mathrm{n}=342 \\
\text { Esquizofrenia, } \\
\text { Ansiedade, } \\
\text { Depressão }\end{array}$ & ISMI & $\begin{array}{l}\text { Mensuração } \\
\text { própria }\end{array}$ & $\begin{array}{l}\text { Percepção de baixo suporte social estava associada a altos níveis } \\
\text { de estigma internalizado. Os autores destacaram que } 0 \text { estigma } \\
\text { internalizado poderia impedir a formação de relações sociais be- } \\
\text { néficas para pacientes com transtorno mental. }\end{array}$ \\
\hline $\begin{array}{l}\text { Brohan et al. } \\
\quad(2010)\end{array}$ & $\begin{array}{l}\text { Descrever o nível de estigma experimen- } \\
\text { tado por usuários de serviço de saúde } \\
\text { mental e sua associação com variáveis } \\
\text { psicossociais. }\end{array}$ & $\begin{array}{l}\mathrm{n}=1229 ; \\
\text { Esquizofrenia/ } \\
\text { Transtorno } \\
\text { Esquizoafetivo/ } \\
\text { Psicose }\end{array}$ & ISMI & $\begin{array}{l}\text { Mensuração } \\
\text { própria }\end{array}$ & $\begin{array}{l}\text { Aumento do número de contatos sociais foi significativamente as- } \\
\text { sociado com a redução do estigma internalizado. }\end{array}$ \\
\hline $\begin{array}{l}\text { Vyavaharkar, et al. } \\
\qquad(2010)\end{array}$ & $\begin{array}{l}\text { Explorar a relação entre estigma relacio- } \\
\text { nado ao HIV, suporte social e depressão } \\
\text { em uma amostra de mulheres que vivem } \\
\text { com HIV/AIDS. }\end{array}$ & 0 & ISS & $\begin{array}{l}\text { MOS-SSS } \\
\text { SSQ }\end{array}$ & $\begin{array}{l}\text { Papel mediador do estigma internalizado entre a avaliação do su- } \\
\text { porte social e depressão. Encontrou-se que o suporte social poderia } \\
\text { afetar o estigma relacionado ao HIV. }\end{array}$ \\
\hline $\begin{array}{l}\text { Person et al. } \\
\text { (2009) }\end{array}$ & $\begin{array}{l}\text { Avaliar as experiências de mulheres que } \\
\text { vivem com a filariose linfática com linfe- } \\
\text { demas na perna. }\end{array}$ & $\begin{array}{l}\mathrm{n}=52 \\
\text { Mulheres } \\
\text { com filariose } \\
\text { linfática }\end{array}$ & Não apresenta & Não apresenta & $\begin{array}{l}\text { A perda das interações sociais devido ao linfedema na perna pare- } \\
\text { ceu contribuir para a internalização do estigma e menor qualidade } \\
\text { de vida. }\end{array}$ \\
\hline $\begin{array}{l}\text { Kalichman et al. } \\
\text { (2009) }\end{array}$ & $\begin{array}{l}\text { Construir e validar a Internalized AIDS } \\
\text {-Related Stigma Scale (IA-RSS) }\end{array}$ & $\begin{array}{l}\mathrm{n}=2394 \\
\text { HIV/AIDS }\end{array}$ & IA-RSS & SSQ & $\begin{array}{l}0 \text { construto de suporte social foi utilizado no estudo como validade } \\
\text { discriminante, encontrando-se uma correlação negativa entre es- } \\
\text { tigma internalizado e suporte social. }\end{array}$ \\
\hline
\end{tabular}




\begin{tabular}{|c|c|c|c|c|c|}
\hline Referência & Objetivo & $\begin{array}{l}\text { Tamanho da } \\
\text { Amostra (n); } \\
\text { População }\end{array}$ & $\begin{array}{l}\text { Instrumentos } \\
\text { Estigma } \\
\text { Internalizado }\end{array}$ & $\begin{array}{l}\text { Instrumentos } \\
\text { Suporte Social }\end{array}$ & Principais Resultados \\
\hline $\begin{array}{c}\text { Sayles et al. } \\
\text { (2008) }\end{array}$ & $\begin{array}{l}\text { Desenvolver e avaliar uma medida mul- } \\
\text { tidimensional de estigma internalizado } \\
\text { do HIV. }\end{array}$ & $\begin{array}{l}\mathrm{n}=202 ; \\
\text { HIV/AIDS }\end{array}$ & $\begin{array}{c}\text { 28-item } \\
\text { measure of } \\
\text { internalized HIV } \\
\text { stigma }\end{array}$ & Fleishman SSS & $\begin{array}{l}0 \text { instrumento de estigma internalizado do HIV se correlacionou ne- } \\
\text { gativamente com a medida de suporte social. Os autores destacam } \\
0 \text { efeito negativo do estigma internalizado nas relações sociais. }\end{array}$ \\
\hline $\begin{array}{l}\text { Simbayi, et al. } \\
\quad(2007)\end{array}$ & $\begin{array}{l}\text { Examinar o estigma internalizado entre } \\
\text { pessoas que vivem com HIV/AIDS na } \\
\text { cidade de Cape Town, na África do Sul. }\end{array}$ & $\begin{array}{c}\mathrm{n}=1063 \\
\text { HIV/ AIDS }\end{array}$ & AIDS-RSS & SSQ & $\begin{array}{l}\text { Correlação negativa entre estigma internalizado e suporte social. } \\
0 \text { estigma internalizado tem um efeito negativo no suporte social, } \\
\text { podendo levar a experiências de angústia psicológica e depressão. }\end{array}$ \\
\hline $\begin{array}{l}\text { Mak, Cheung et al. } \\
\text { (2007) }\end{array}$ & $\begin{array}{l}\text { Testar um modelo de explicação do estig- } \\
\text { ma internalizado. }\end{array}$ & $\begin{array}{l}n=150 \\
\text { HIV/AIDS }\end{array}$ & $\begin{array}{l}\text { Mensuração } \\
\text { própria }\end{array}$ & MOS-SSS & $\begin{array}{l}\text { Correlações negativas significativas entre estigma internalizado e } \\
\text { suporte social. Pessoas que vivem com HIV/AIDS são mais prová- } \\
\text { veis de perceberem menos apoio por parte dos outros. }\end{array}$ \\
\hline $\begin{array}{l}\text { Ersoy \& Varan } \\
\quad(2007)\end{array}$ & $\begin{array}{l}\text { Avaliar a confiabilidade e validade da } \\
\text { versão turca da Internalized Stigma of } \\
\text { Mental IIIness Scale (ISMI) em pacientes } \\
\text { com transtorno mental. }\end{array}$ & $\begin{array}{l}\mathrm{n}=203 \\
\text { Transtorno } \\
\text { mental }\end{array}$ & ISMI & MSPSS & $\begin{array}{l}\text { Correlação negativa entre estigma internalizado e suporte social. A } \\
\text { redução das relações sociais devido ao transtorno mental podem } \\
\text { contribuir para a internalização do estigma. }\end{array}$ \\
\hline
\end{tabular}

ISMI = Internalized Stigma of Mental IIIness; IA-RSS = Internalized AIDS-Related Stigma Scale; AIDS-RSS = AIDS-Related Stigma Scale.

SSRS = Social Support Rating Scale; MSPSS = Multidimensional Scale of Perceived Social Support; SSQ = Social Support Questionnaire; MOS-SSS = Medical Outcomes Study Social Support Survey; Fleishman SSS = Fleishman Social Support Scale

Principais limitações e direcionamentos para pesquisas futuras. Dentre os estudos analisados, cinco destacaram como principal limitação o pequeno tamanho da amostra, dificultando a generalização dos resultados (Adewuya et al., 2011; Cerit et al., 2012; Lv et al., 2012; Sayles et al., 2008; Zafran et al., 2012). Ainda com relação à amostra, dois estudos destacaram o viés de tratamento, argumentando que a percepção de estigma internalizado e suporte social para participantes engajados no tratamento pode diferir daqueles que não estão em situação de tratamento (Brohan et al., 2010; Zafran et al., 2012). Outras limitações importantes se referiram ao desenho transversal dos estudos, que não permite o estabelecimento de uma relação causal entre as variáveis investigadas (Adewuya et al., 2011; Brohan et al., 2010; Vyavaharkar et al., 2010), e a amostragem por conveniência, comprometendo a validade externa dos estudos (Lv et al., 2012; Sayles et al., 2008; Simbayi et al., 2007).

Como direcionamentos para pesquisas futuras destacou-se como principal a necessidade do desenvolvimento de intervenções apropriadas para prevenir o estigma e reduzir seus efeitos negativos para o indivíduo (Adewuya et al., 2011; Brohan et al., 2010; Peltzer \& Ramlagan, 2011; Person et al., 2009; Simbayi et al., 2007). Para isso, os autores ressaltam como alternativa o investimento no desenvolvimento de contatos sociais (Brohan et al., 2010), e na adaptação e ajustamento social (Simbayi et al., 2007). Mak et al. (2007) ressalta ainda a importância de se investigar as dimensões culturais que podem influenciar as percepções sobre a condição de saúde, e a necessidade da realização de mais estudos que avaliem o processo de internalização do estigma no contexto das relações sociais.

\section{Discussão}

O presente estudo buscou sintetizar os resultados de estudos empíricos acerca da relação entre estigma internalizado e suporte social, e as implicações dessa relação para a saúde. Com base nos resultados encontrados, foi possível observar uma associação negativa entre essas variáveis, uma vez que maiores níveis de estigma internalizado estiveram relacionados a menores níveis de suporte social. Essa relação sugere que o estigma internalizado possui um efeito negativo nas dimensões de vida do indivíduo à medida que o afastamento do convívio social, além de restringir as redes sociais e as oportunidades de vida (Adewuya et al., 2011), traz implicações para o contexto da saúde, como a piora na qualidade de vida (Peltzer \& Ramlagan, 2011; Person et al., 2009), experiências de angústia psicológica (Person et al., 2009; Simbayi et al., 2007) e presença de sintomas depressivos (Peltzer \& Ramlagan, 2011; Simbayi et al., 2007; Vyavaharkar et al., 2010). Independente do diagnóstico, a depressão se mostrou como o principal indicador negativo de saúde mental associado ao estigma internalizado, embora nem sempre seja possível saber ao certo a direção dessa associação já que alguns estudos têm mostrado que o estigma internalizado aumenta os sintomas depressivos, enquanto que outros têm sugerido que a depressão contribui para um maior nível de estigma internalizado (Ritsher et al., 2003; Vyavaharkar et al., 2010).

A comorbidade da depressão com outras condições de saúde consiste em uma importante implicação para o tratamento, uma vez que tende a agravar o estado de saúde, além de ter um impacto negativo na qualidade de vida. Além disso, a presença de sintomas depressivos contribui para o isolamento e evitação social, principalmente daqueles que são significativos em promover assistência e suporte emocional, podendo inclusive interferir na adesão ao tratamento 
da condição primária (DiMatteo, Lepper, \& Croghan, 2000; Moussavi et al., 2007).

Estudos anteriores discutiram ainda que a internalização do estigma acarreta em diversas dificuldades para o tratamento, incluindo não só a baixa adesão (Alvidrez, Snowden, \& Kaiser, 2008; Kalichman \& Grebler, 2010; Tsang, Fung, \& Chung, 2010), como também o agravamento dos sintomas (Ritsher \& Phelan, 2004; Yanos, et al., 2008), inibição para a prontidão para a mudança (Tsang et al., 2010), além de reforçar as expectativas pessoais de rejeição e discriminação (Alvidrez et al., 2008). Assim, o suporte social tem sido um construto descrito como um potencial recurso de enfrentamento ao estigma, na medida em que a disponibilidade e a satisfação com o suporte parecem afetar o ajustamento das pessoas (DiMatteo, 2004; Seidl \& Tróccoli, 2006), auxiliando na reestruturação e manutenção das relações sociais (Brohan et al., 2010; Lv et al., 2012; Zafran et al., 2012), além de influenciar positivamente a progressão e a recuperação de uma doença, no que se refere à adoção de comportamentos saudáveis (Mueller et al., 2006; Schwarzer, Knoll, \& Rieckmann, 2003).

A presença de um suporte social pode contribuir significativamente para diminuir o estresse psicológico, os sintomas depressivos e as experiências de estigma (Vyavaharkar et al., 2010). Essas contribuições evidenciam a necessidade de se considerar construtos como o estigma internalizado e o suporte social ao longo do tratamento da condição, além de auxiliar na elaboração de intervenções apropriadas para o enfrentamento ao estigma de forma a diminuir o seu impacto negativo na vida dos indivíduos (Adewuya et al., 2011; Brohan et al., 2010; Peltzer \& Ramlagan, 2011; Person et al., 2009; Simbayi et al., 2007; Vyavaharkar et al., 2010).

Fatores sociodemográficos também estiveram associados aos níveis de estigma internalizado e de suporte social, sendo esses resultados consistentes com a literatura que aponta que desemprego, baixa renda e menores níveis de escolaridade atuam como preditores de maiores níveis de estigma internalizado (Li, Lee, Thammawijaya, Jiraphongsa, \& Rotheram-Borus, 2009; Werner, Stein-Shvachman, \& Heinik, 2009). O longo curso da doença aumenta a probabilidade de se desenvolver algumas debilidades em função da condição, um funcionamento pobre que acaba por contribuir para o desemprego e baixa renda, contribuindo para o aumento do estigma internalizado (Adewuya et al., 2011). Em relação ao gênero, observou-se que ser do sexo masculino estava relacionado a maiores níveis de estigma internalizado. Entretanto, outros estudos revelaram que mulheres apresentam maiores níveis de estigma (Brohan et al., 2010; Werner et al., 2009), o que sugere a realização de estudos que investiguem o papel da variável sexo na internalização do estigma.

Por outro lado, ser mulher esteve associado com maiores níveis de suporte social (Cerit et al., 2012; Simbayi et al., 2007), visto que a literatura aponta que mulheres normalmente possuem mais amigos próximos do que os homens, e tendem a desenvolver relações mais íntimas, embora homens tendam a se engajar em grandes grupos. Além disso, as mulheres fornecem mais apoio emocional tanto para homens quanto para mulheres, conseguindo maior ajuda em troca (Schwarzer et al., 2003).

A ausência de uma definição de suporte social pelos estudos revela uma confusão conceitual do construto, uma vez que o termo suporte social é geralmente usado em um sentido amplo, incluindo os conceitos de rede social e integração social que assumem posições teóricas distintas, o que influencia diretamente na escolha dos instrumentos de mensuração. Assim, a falta de clareza do construto não permite a compreensão acerca de quais dimensões de suporte social estão sendo avaliadas (Schwarzer et al., 2003). Por outro lado, o conceito de estigma internalizado foi definido de forma convergente, sendo Patrick Corrigan o principal autor citado. Considerando que o instrumento de medida de estigma internalizado mais utilizado foi a escala Internalized Stigma of Mental Illness (ISMI) (Ritsher et al., 2003), é possível destacar que os autores enfatizam variáveis como o sigilo da condição, desvalorização percebida, vergonha e exclusão social como processos essenciais para a internalização do estigma, diferente do proposto por Corrigan que concebe o estigma internalizado em termos da relação com autoestima e autoeficácia (Corrigan et al., 2006; Soares, Nery, Silveira, Noto, \& Ronzani, 2011) . Embora tenham sido propostas definições similares acerca do construto, observa-se a presença de bases conceituais distintas o que acarreta em dificuldades para a comparação dos resultados entre os estudos (Link \& Phelan, 2001).

Alguns problemas metodológicos puderam ser observados, e foram apontados como limitações das pesquisas. O delineamento dos estudos analisados é um fator que merece destaque, já que a maioria $(n=11)$ optou por um desenho transversal. A realização de estudos longitudinais seria de grande importância para a compreensão da relação entre estigma internalizado e suporte social, e para a avaliação das consequências dessa relação a longo prazo. Considerando que apenas três estudos relataram a estratégia de amostragem probabilística para a seleção dos participantes, há uma dificuldade de generalização dos resultados para a população geral, observada também pela utilização de amostras pequenas. O viés do tratamento também pode ser considerado como uma limitação importante, uma vez que pode influenciar diretamente nas percepções de suporte social e de estigma internalizado de participantes que estão em tratamento.

Tendo em vista os estudos que compuseram a amostra deste artigo, observa-se que apenas três tipos de condições de saúde foram investigados, sendo elas o transtorno mental ( $n$ $=6)$, o HIV/AIDS $(n=6)$ e a filariose linfática $(n=1)$. Van Brakel (2006) argumenta que as consequências do estigma têm se mostrado similares entre diversas condições de saúde, no entanto, considera-se fundamental a realização de estudos com populações distintas visto que o impacto da relação entre estigma internalizado e suporte social pode se apresentar de diferentes formas de acordo com o grau de aceitação social de determinada condição (Link \& Phelan, 2001; Mak et al., 2007). Estudos apontam que nos casos em que o indivíduo é considerado como responsável pela sua condição, como no abuso e dependência de substâncias, por exemplo, a rejeição 
social é particularmente forte, sendo que as experiências de discriminação têm um impacto negativo na disponibilidade de suporte social, contribuindo para a deterioração da saúde na medida em que se constitui como uma potencial barreira para o acesso ao cuidado (Ahern, Stuber, \& Galea, 2007; Silveira, Martins, \& Ronzani, 2009; Simbayi et al., 2007).

Considerando a importância do tema, sugere-se que mais estudos que avaliem a relação entre suporte social e estigma internalizado entre condições de saúde distintas sejam realizados, uma vez que apenas treze estudos foram encontrados. Entretanto, a presente revisão sistemática traz duas importantes contribuições para a literatura. A primeira se refere ao impacto negativo da internalização do estigma para resultados de saúde, principalmente de pacientes com transtorno mental e de pessoas que vivem com HIV/AIDS, e a necessidade de intervenções e políticas voltadas para a redução do estigma e dos danos associados. A segunda contribuição se refere ao construto de suporte social como uma possível estratégia de enfrentamento ao estigma internalizado, considerando o seu efeito protetor ao amenizar as consequências negativas de eventos estressantes sobre o bem-estar físico e psicológico (Seidl \& Tróccoli, 2006), além de favorecer o estabelecimento de relações saudáveis que promovam a reintegração social e melhor qualidade de vida (Vyavaharkar et al., 2010; Zafran et al., 2012).

\section{Limitações}

Algumas limitações precisam ser consideradas na interpretação dos resultados do presente estudo. Como o objetivo do estudo consistiu em avaliar a relação entre estigma internalizado e suporte social e o impacto dessa relação para a saúde, aspectos sociais relacionados a categorias sociais como renda, escolaridade, papel do gênero, etnia e orientação sexual não foram investigados. Em segundo lugar, conceitos como 'rede social' e 'integração social' por serem considerados distintos do conceito de suporte social não foram utilizados como palavras-chave para a busca dos artigos. Da mesma forma, conceitos como vergonha internalizada e homofobia internalizada foram excluídos por representarem apenas dimensões isoladas do estigma internalizado ou fazerem referência a condições sociais, e não de saúde. A terceira limitação se refere à variedade de instrumentos de mensuração de estigma internalizado e suporte social, o que sugere que diferentes domínios dos construtos tenham sido avaliados, requerendo maior atenção na comparação entre os resultados.

\section{Agradecimentos}

Agradecemos à Coordenação de Aperfeiçoamento de Pessoal de Nível Superior (CAPES) pela concessão da bolsa de mestrado. Ao Conselho Nacional de Desenvolvimento Científico e Tecnológico ( $\mathrm{CNPq}$ ) e à Fundação de Amparo à Pesquisa do Estado de Minas Gerais (FAPEMIG) pelo apoio financeiro à pesquisa.

\section{Referências}

Adewuya, A. O., Owoeye, A. O., Erinfolami, A. O., \& Ola, B. A. (2011). Correlates of self-stigma among outpatients with mental illness in Lagos, Nigeria. International Journal of Social Psychiatry, 57(4), 418-427. doi: 10.1177/0020764010363522

Ahern, J., Stuber, J., \& Galea, S. (2007). Stigma, discrimination and the health of illicit drug users. Drug and Alcohol Dependence, 88, 188-196. doi: 10.1016/j.drugalcdep.2006.10.014

Alvidrez, J., Snowden, L. R., \& Kaiser, D. M. (2008). The experience of stigma among black mental health consumers. Journal of Health Care for the Poor and Underserved, 19(3), 874-893. doi: 10.1353/hpu.0.0058

Brock, D., Sarason, I., Sarason, B., \& Pierce, G. (1996). Simultaneous assessment of perceived global and relationship-specific support. Journal of Social and Personal Relationships, 13, 143-152.

Brohan, E., Elgie, R., Sartorius, N., Thornicroft, G., \& Grp, G. A.-E. S. (2010) Self-stigma, empowerment and perceived discrimination among people with schizophrenia in 14 European countries: The GAMIAN-Europe study. Schizophrenia Research, 122(1-3), 232-238. doi: 10.1016/j. schres.2010.02.1065

Cerit, C., Filizer, A., Tural, Ü., \& Tufan, A. E. (2012). Stigma: A core factor on predicting functionality in bipolar disorder. Comprehensive Psychiatry, 53(5), 484-489. doi: 10.1016/j.comppsych.2011.08.010

Chou, C. C., Robb, J. L., Clay, M. C., \& Chronister, J. A. (2013). Social support as a mediator between internalized stigma and coping behaviors of individuals with substance abuse issues. Rehabilitation Research, Policy, and Education, 27(3), 104-107. doi: 10.1891/2168-6653.27.2.104

Cochrane Collaboration. (2013). Systematic Review - Glossary The Cochrane Collaboration. Recuperado de http://www.cochrane.org/glossary.

Corrigan, P. W., \& Watson, A. C. (2002). The paradox of self-stigma and mental illness. Clinical Psychology-Science and Practice, 9(1), 35-53.

Corrigan, P. W., Morris, S., Larson, J., Rafacz, J., Wassel, A., Michaels, P., ... Rüsch, N. (2010). Self-stigma and coming out about one's mental illness. Journal of Community Psychology, 38(3), 259-275. doi: 10.1002/ jcop. 20363

Corrigan, P. W., Sokol, K. A., \& Rüsch, N. (2011). The impact of self-stigma and mutual help programs on the quality of life of people with serious mental illnesses. Community Mental Health Journal, 49(1), 1-6. doi: 10.1007/ s10597-011-9445-2.

Corrigan, P. W., Watson, A. C., \& Barr, L. (2006). The self-stigma of mental illness: Implications for self-esteem and self-efficacy. Journal of Social and Clinical Psychology, 25(8), 875-884. doi:10.1521/jscp.2006.25.8.875

DiMatteo, M. R. (2004). Social support and patient adherence to medical treatment: A meta-analysis. Health Psychology, 23(2), 207-218. doi:10.1037/0278-6133.23.2.207

DiMatteo, M. R., Lepper, H. S., \& Croghan, T. W. (2000). Depression is a risk factor for noncompliance with medical treatment: Meta-analysis of the effects of anxiety and depression on patient adherence. Archives of Internal Medicine, 160(14), 2101-2107. doi:10.1001/archinte.160.14.2101

Ersoy, M. A., \& Varan, A. (2007). Reliability and validity of the Turkish version of the Internalized Stigma of Mental Illness Scale. Turk Psikiyatri Dergisi, 18(2), 163-171.

Fife, B. L., \& Wright, E. R. (2000). The dimensionality of stigma: A comparison of its impact on the self of persons with HIV/AIDS and cancer. Journal of Health and Social Behavior, 48, 50-67. doi: 10.2307/2676360

Kalichman, S. C., \& Grebler, T. (2010). Stress and poverty predictors of treatment adherence among people with low-literacy living with HIV/AIDS. Psychosomatic Medicine, 72(8), 810-816. doi: 10.1097/ PSY.0b013e3181f01be3

Kalichman, S. C., Simbayi, L. C., Cloete, A., Mthembu, P. P., Mkhonta, R. N., \& Ginindza, T. (2009). Measuring AIDS stigmas in people living with HIV/ AIDS: the Internalized AIDS-Related Stigma Scale. AIDS Care, 21(1), 8793. doi: $10.1080 / 09540120802032627$

Li, L., Lee, S. J., Thammawijaya, P., Jiraphongsa, C., \& Rotheram-Borus, M. J. (2009). Stigma, social support, and depression among people living with 
HIV in Thailand. Aids Care-Psychological and Socio-Medical Aspects of Aids/ Hiv, 21(8), 1007-1013. doi: 10.1080\%2F09540120802614358

Link, B. G., \& Phelan, J. C. (2001). Conceptualizing Stigma. Annual Review of Sociology, 27, 363 - 385. doi: 10.1146/annurev.soc.27.1.363

Link, B. G., \& Phelan, J. C. (2006). Stigma and its public health implications. Lancet, 367, 528-529. doi:10.1016/S0140-6736(06)68184-1

Lv, Y., Wolf, A., \& Wang, X. (2013). Experienced stigma and self-stigma in Chinese patients with schizophrenia. General Hospital Psychiatry, 35(1), 83-88. doi: 10.1016/j.genhosppsych.2012.07.007.

Mak, W. W. S., Cheung, R. Y. M., Law, R. W., Woo, J., Li, P. C. K., \& Chung, R. W. Y. (2007). Examining attribution model of self-stigma on social support and psychological well-being among people with HIV+/AIDS. Social Science \& Medicine, 64(8), 1549-1559. doi: 10.1016/j.socscimed.2006.12.003

Measelle, J. R., Stice, E., \& Springer, D. W. (2006). A prospective test of the negative affect model of substance abuse: Moderating effects of social support. Psychology of Addictive Behaviors, 20(3), 225-233. doi: 10.1037\%2F0893-164X.20.3.225

Michalak, E., Livingston, J. D., Hole, R., Suto, M., Hale, S., \& Haddock, C. (2011). 'It's something that I manage but it is not who I am': Reflections on internalized stigma in individuals with bipolar disorder. Chronic Illness, 7(3), 209-224. doi: 10.1177/1742395310395959

Mickelson, K. D. (2001). Perceived stigma, social support, and depression. Personality and Social Psychology Bulletin, 27(8), 1046-1056. doi: 10.1177/0146167201278011

Moussavi, S., Chatterji, S., Verdes, E., Tandon, A., Patel, V., \& Ustun, B. (2007). Depression, chronic diseases, and decrements in health: results from the World Health Surveys. Lancet, 370, 851-58. doi: 10.1016/s01406736(07)61415-9

Mueller, B, Nordt, C., Lauber, C., Rueesch, P., Meyer, P. C., \& Roessler, W. (2006). Social support modifies perceived stigmatization in the first years of mental illness: A longitudinal approach. Social Science \& Medicine, 62, 39-49. doi:10.1016/j.socscimed.2005.05.014

Peltzer, K., \& Ramlagan, S. (2011). Perceived stigma among patients receiving antiretroviral therapy: a prospective study in KwaZulu-Natal, South Africa. Aids Care-Psychological and Socio-Medical Aspects of Aids/Hiv, 23(1), 60-68. doi: 10.1080/09540121.2010.498864

Person, B., Bartholomew, L. K., Gyapong, M., Addiss, D. G., \& van den Borne, B. (2009). Health-related stigma among women with lymphatic filariasis from the Dominican Republic and Ghana. Social Science \& Medicine, 68(1), 30-38. doi: 10.1016/j.socscimed.2008.09.040

Ritsher, J. B., \& Phelan, J. C. (2004). Internalized stigma predicts erosion of morale among psychiatric outpatients. Psychiatry Research, 129(3), $257-$ 265. doi: 10.1016/j.psychres.2004.08.003

Ritsher, J. B., Otilingam, P. G., \& Grajales, M. (2003). Internalized stigma of mental illness: psychometric properties of a new measure. Psychiatry Research, 121, 31-49. doi:10.1016/j.psychres.2003.08.008

Sayles, J. N., Hays, R. D., Sarkisian, C. A., Mahajan, A. P., Spritzer, K. L., \& Cunningham, W. E. (2008). Development and psychometric assessment of a multidimensional measure of internalized HIV stigma in a sample of HIV-positive adults. Aids and Behavior, 12(5), 748-758. doi: 10.1007/ s10461-008-9375-3

Schwarzer, R., \& Knoll, N. (2007) Functional roles of social support within the stress and coping process: A theoretical and empirical overview. International Journal of Psychology, 42(4), 243-252. doi: 10.1080/00207590701396641

Schwarzer, R., Knoll, N., \& Rieckmann, N. (2003). Social support. In A. Kaptein \& J. Weinman (Orgs.), Introduction to health psychology (pp. 158182). Oxford: Blackwell.

Seidl, E. M. F, \& Tróccoli, B. T. (2006). Desenvolvimento de escala para avaliação do suporte social em HIV/AIDS. Psicologia: Teoria e Pesquisa, 22(3), $317-$ 326. doi: 10.1590/S0102-37722006000300008

Sibitz, I., Amering, M., Unger, A., Seyringer, M. E., Bachmann, A., Schrank, B., ... Woppmann, A. (2011). The impact of the social network, stigma and empowerment on the quality of life in patients with schizophrenia. European Psychiatry, 26(1), 28-33. doi: 10.1016/j.eurpsy.2010.08.010

Silveira, P. S., Martins, L. F., \& Ronzani, T. M. (2009). Moralização sobre o uso de álcool entre agentes comunitários de saúde. Psicologia Teoria e Prática, 11, 62-75. doi: 10.1590/S1413-294X2010000100005

Simbayi, L. C., Kalichman, S., Strebel, A., Cloete, A., Henda, N., \& Mqeketo, A. (2007). Internalized stigma, discrimination, and depression among men and women living with HIV/AIDS in Cape Town, South Africa. Social Science \& Medicine, 64(9), 1823-1831. doi: 10.1016/j.socscimed.2007.01.006

Soares, R. G., Nery, F. C., Silveira, P. S., Noto, A. R., \& Ronzani, T. M. (2011). A mensuração do estigma internalizado: Revisão sistemática da literatura. Psicologia em Estudo, 16(4), 635-645. doi: 10.1590/S141373722011000400014

Tsang, H. W. H., Fung, K. M. T., \& Chung, R. C. K. (2010). Self-stigma and stages of change as predictors of treatment adherence of individuals with schizophrenia. Psychiatry Research, 180(1), 10-15. doi: 10.1016/j. psychres.2009.09.001

VanBrakel, W. H. (2006). Measuring health-related stigma - A literature review. Psychology, Health \& Medicine, 11(3), 307-334.

Vyavaharkar, M., Moneyham, L., Corwin, S., Saunders, R., Annang, L., \& Tavakoli, A. (2010). relationships between stigma, social support, and depression in hiv-infected African American women living in the rural southeastern United States. Janac-Journal of the Association of Nurses in Aids Care, 21(2), 144-152. doi: 10.1016/j.jana.2009.07.008

Walton, M. A., Blow, F. C., Bingham, C. R., \& Chermack, S. T. (2003). Individual and social/environmental predictors of alcohol and drug use 2 years following substance abuse treatment. Addictive Behaviors, 28, 627-642. doi:10.1016/S0306-4603(01)00284-2

Watson, A. C., Corrigan, P., Larson, J. E., \& Sells, M. (2007). Self-stigma in people with mental illness. Schizophrenia Bulletin, 33(6), 1312-1318. doi: $10.1093 / \mathrm{schbul} / \mathrm{sbl076}$

Werner, P., Stein-Shvachman, I., \& Heinik, J. (2009). Perceptions of self-stigma and its correlates among older adults with depression: A preliminary study. International Psychogeriatrics, 21(6), 1180-1189. doi: 10.1017/ S1041610209990470

Yanos, P. T., Roe, D., Markus, K., \& Lysaker, P. H. (2008). Pathways between internalised stigma and outcomes related to recovery in schizophrenia spectrum disorders. Psychiatric Services, 59, 1437-1442. doi: 10.1176/ appi.ps.59.12.1437

Zafran, H., Tallant, B., \& Gelinas, I. (2012). A first-person exploration of the experience of academic reintegration after first episode psychosis. International Journal of Psychosocial Rehabilitation, 16(1), 29-46. 
Gabriela Correia Lubambo Ferreira, Mestre em Psicologia pela Universidade Federal de Juiz de Fora (UFJF), é pesquisadora do Centro de Referência em Pesquisa, Intervenção e Avaliação em Álcool e Drogas (CREPEIA) da UFJF. Endereço para correspondência: Av. Joaquim Dutra, 169/102. Bairro Monte Verde. São Lourenço, Minas Gerais - MG. CEP: 37.470.000. Telefone: (35) 8882-0990. E-mail: gabicorreia@ gmail.com

Pollyanna Santos da Silveira, Mestre em Psicologia pela Universidade Federal de Juiz de Fora (UFJF), é Doutoranda em Ciências da Saúde pelo Departamento de Psicobiologia da Universidade Federal de São Paulo (UNIFESP) vinculado à Disciplina de Medicina e Sociologia de Abuso de Drogas (DIMESAD) e Pesquisadora do Centro de Referência em Pesquisa, Intervenção e Avaliação em Álcool e Drogas (CREPEIA) da UFJF. E-mail: pollyannassilveira@gmail.com

Ana Regina Noto, Doutora em Psicobiologia pela Universidade Federal de São Paulo (UNIFESP), é Professora Adjunta e Chefe da DIMESAD (Disciplina de Medicina e Sociologia do Abuso de Drogas) do Departamento de Psicobiologia da Universidade Federal de São Paulo (UNIFESP) e Coordenadora do Núcleo de Pesquisa sobre Saúde e Uso de Substâncias (NEPSIS) e do Centro Regional de Referência em Crack e outras Drogas - CRR-DIMESAD-UNIFESP. E-mail: anareginanoto@gmail.com

Telmo Mota Ronzani, Doutor em Ciências da Saúde pela Universidade Federal de São Paulo (UNIFESP) e Pós-Doutor em Ciências da Saúde pela Universidade de São Paulo (USP) e University of Coneecticut Health Center (UCHC), é Professor da Universidade Federal de Juiz de Fora (UFJF) e professor e Coordenador do Programa de Pós-Graduação strito senso em Psicologia da UFJF. Bolsista de Produtividade CNPq. E-mail: tm.ronzani@gmail.com 